\section{8*BRC}

Graduate Students, Medical Biomedical and Health Sciences

\title{
Production of active long lasting CNGRC-CPG2 fusion protein using PEGylation to be used in Ligand Directed Cancer Therapy
}

\author{
Layla Al-Mansoori1,2, Philip Elsinga ${ }^{2}$ and Sayed K. Goda ${ }^{3}$ \\ ${ }_{1}^{1}$ Biomedical Research Center-Qatar University, QATAR.
}

2 Department of Nuclear Medicine and Molecular Imaging, University of Medical Center Groningen "UMCG", University of Groningen- Netherlands.

${ }^{3}$ Cairo University, Faculty of Science, Chemistry Department, Giza, Egypt

\section{ABSTRACT}

Aminopeptidase $N(A P N)$ is one of the important enzymes highly expressed in metastatic cancers, thus employed as a marker to target tumor cells. CPG2-CNGRC fusion protein is produced to target high APN expressing cancer cells, which with the prodrug results in high toxic effect. Since PEGylation of CPG2 has shown an improved favorable in vitro stability and immunotoxicity, we performed a site-directed PEGylation (thiol group directed) of the CPG2-CNGRC fusion protein and group directed) of the CPG2-CNGRC fusion protein and protein's therapeutic efficacy. CPG2 kinetic activity was substantially enhanced following PEGylation of the single fusion protein (PEG CPG2-CNGRC). The binding affinity of the produced PEGylated fusion proteins to their cellular marker (APN) was notably reduced in case of the double fusion protein compared with non-PEGylated ones. Moreover, the cytotoxic effect of methotrexate and ZD2767P (prodrug) in association of the PEGylated fusion proteins was investigated and found that the cytotoxic effect of prodrug with PEGylated single fusion protein was improved significantly (low cell survival). Similar finding was found following MTX treatment where lower binding and kinetic activity of the PEGylated double fusion proteins resulted in higher MTX toxic effect (lower cell survival) in comparison with the non-PEGylated double fusion protein. Thus, although PEGylation is known for its usually favorable effect on the protein/drug pharmacodynamics, our results indicated that with our different fusion proteins (single and double fusion proteins) PEGylation did not improve it similarly.

\section{INTRODUCTION}

The use of anticancer agents have been facing several drawbacks as their poor solubility, short in vivo half-life, low specificity to the tumor cells resulting in low therapeutic efficacy and serious side effects (1). PEGylation (conjugation of protein and/or drug with PolyEthylene Glycol "PEG" polymer) is well known approach widely used lately to improve pharmacokinetics and therapeutic properties of peptides and drugs used in cancer therapy (2-4). We previously succeeded to conjugate PEG polymer to the therapeutic enzyme glucarbidase (carboxypeptidase G2 (CPG2)) and generated a PEGylated biobetter glucarbidase variant (5). Moreover, our recent study presented a developed fusion protein using the aminopeptidase $N$ (APN) binding peptide (CNGRC) conjugated to CPG2 targeting APN known as a target for meastatic cancers (6).

In the current study we combined the two approaches and applied PEGylation to the previously generated fusion proteins (single"X-CPG2" and double "X-CPG2-X") producing PEGylated fusion proteins (PEG single "PEG X-CPG2" and PEG "PEG $X$ CPG2-X"double fusion proteins). We assessed the effectiveness of PEGylated fusion proteins as potential therapeutic compounds used in combination of a prodrug for targeted cancer therapy.

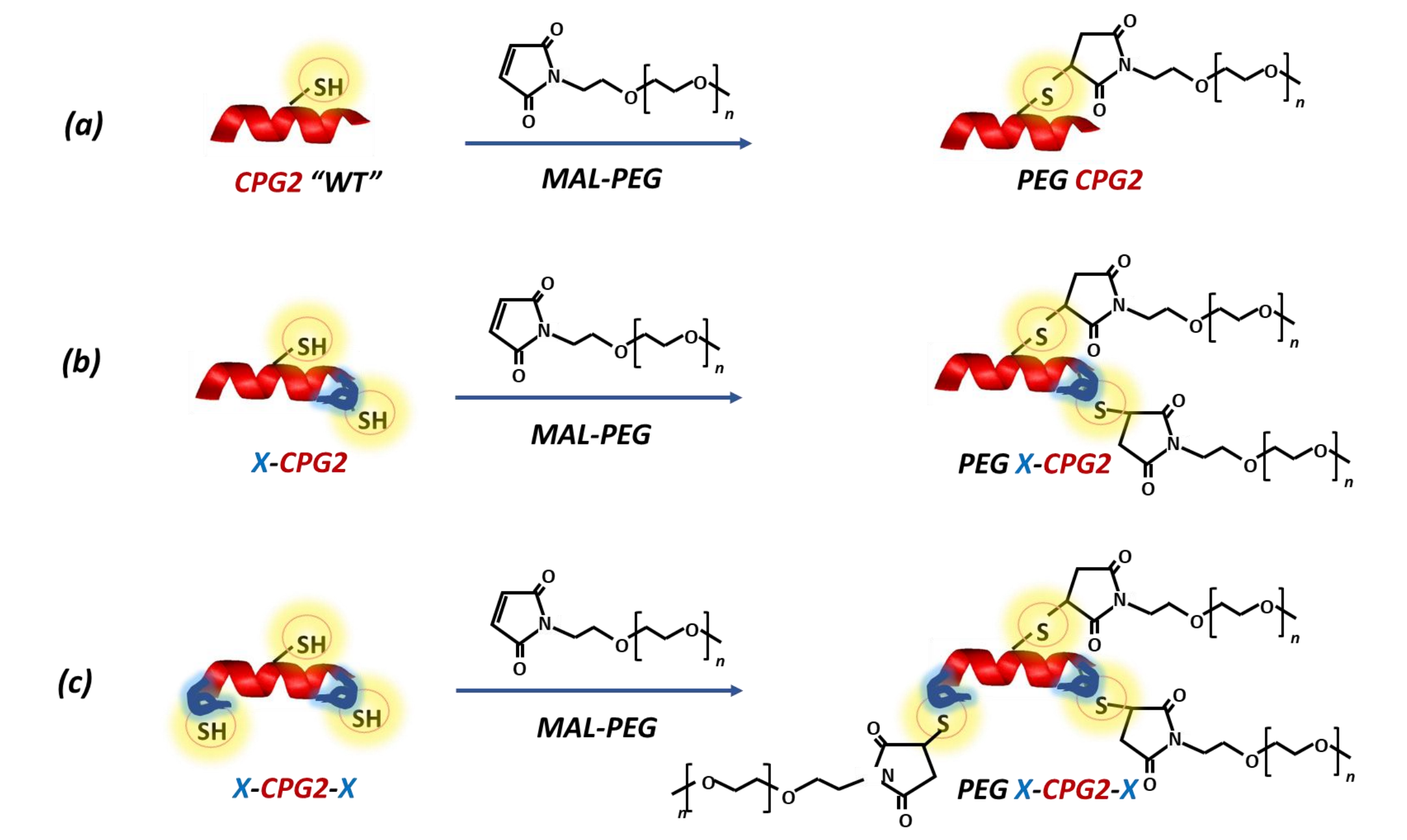

Fig. 1 Site specific PEGylation of CPG2 at Cysteine moiety (-SH). MaleimidePEG group (MAL-PEG) reacts specifically with the $-\mathrm{SH}$ residues in the protein Carboxypeptidase G2 "CPG2" forming a PEG-CPG2 conjugate. (a) Reaction of WT CPG2 which has one Cys. Group with MAL-PEG. (b) Reaction of single
CPG2 fusion protein X-CPG2 (CNGRC- peptide "blue" fused at N terminal)

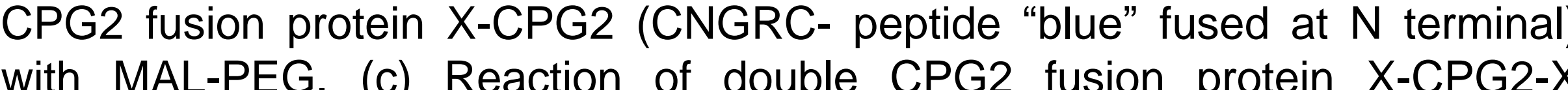
with MAL-PEG. (c) Reaction of double CPG2 fusion protein X-CPG2-X CNGRC.

\section{INTRODUCTION}

Preparation, identification and structural analysis of the PEGylated CPG2 fusion proteins:

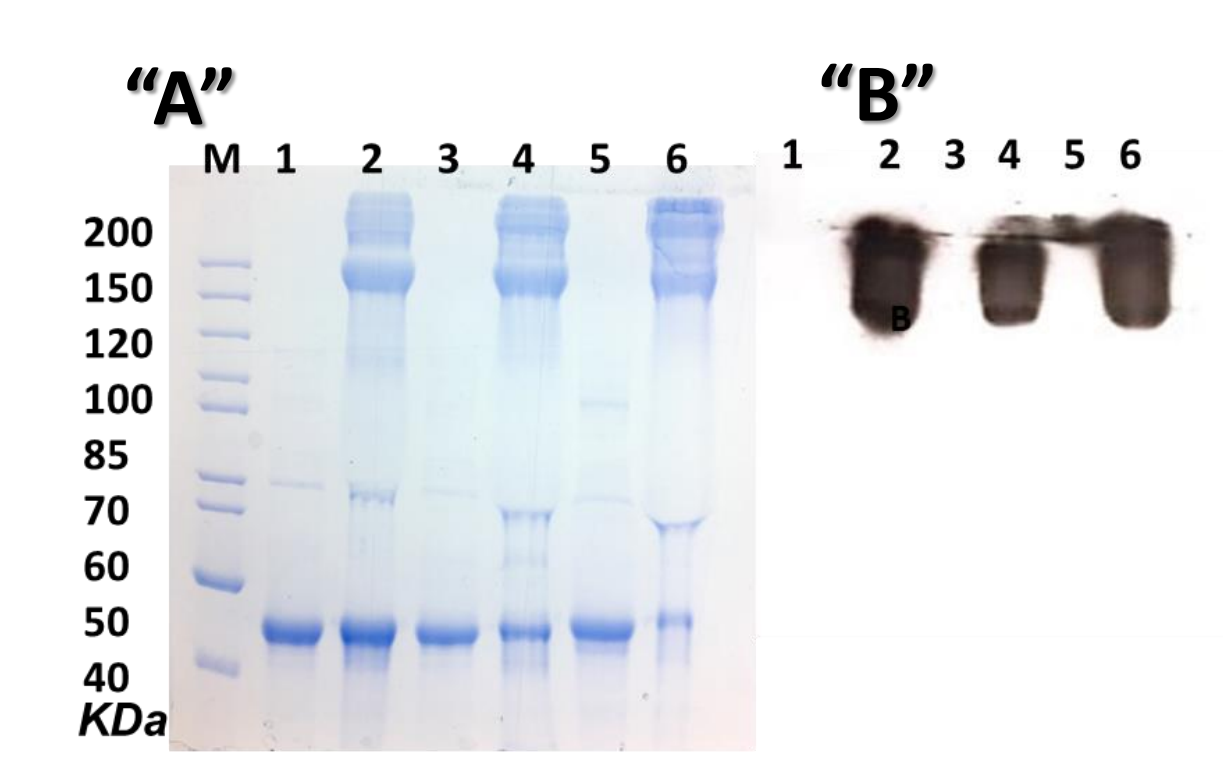

Fig. 2 Identification of
PEGylated CPG2 proteins. The PEGylated CPG2 proteins. The
resulting PEG- CPG2 fusion resulting PEG- CPG2 fusion
proteins (WT "PEG-CPG2",

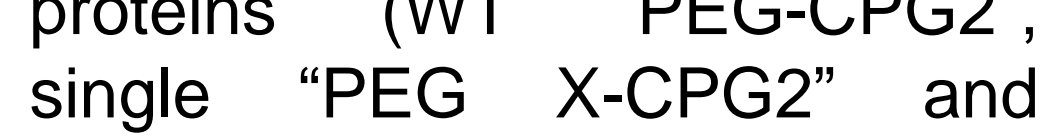
double "PEG X-CPG2-X" fusion proteins) were separated on
SDS-PAGE and either (A) stained with Coomassie brilliant Blue or (B) western blot with anti-PEG antibody. Lanes 1, 3 and 5 show the whereas lanes 24 and 6 show the bands for PEGylated $C P G 2$ proteins (PEG CPG2, PEG X-CPG2 and PEG X-CPG2-X respectively). PEG: polyethylene glycol, CPG2: Carboxypeptidase G2, X: CNGRC peptide.

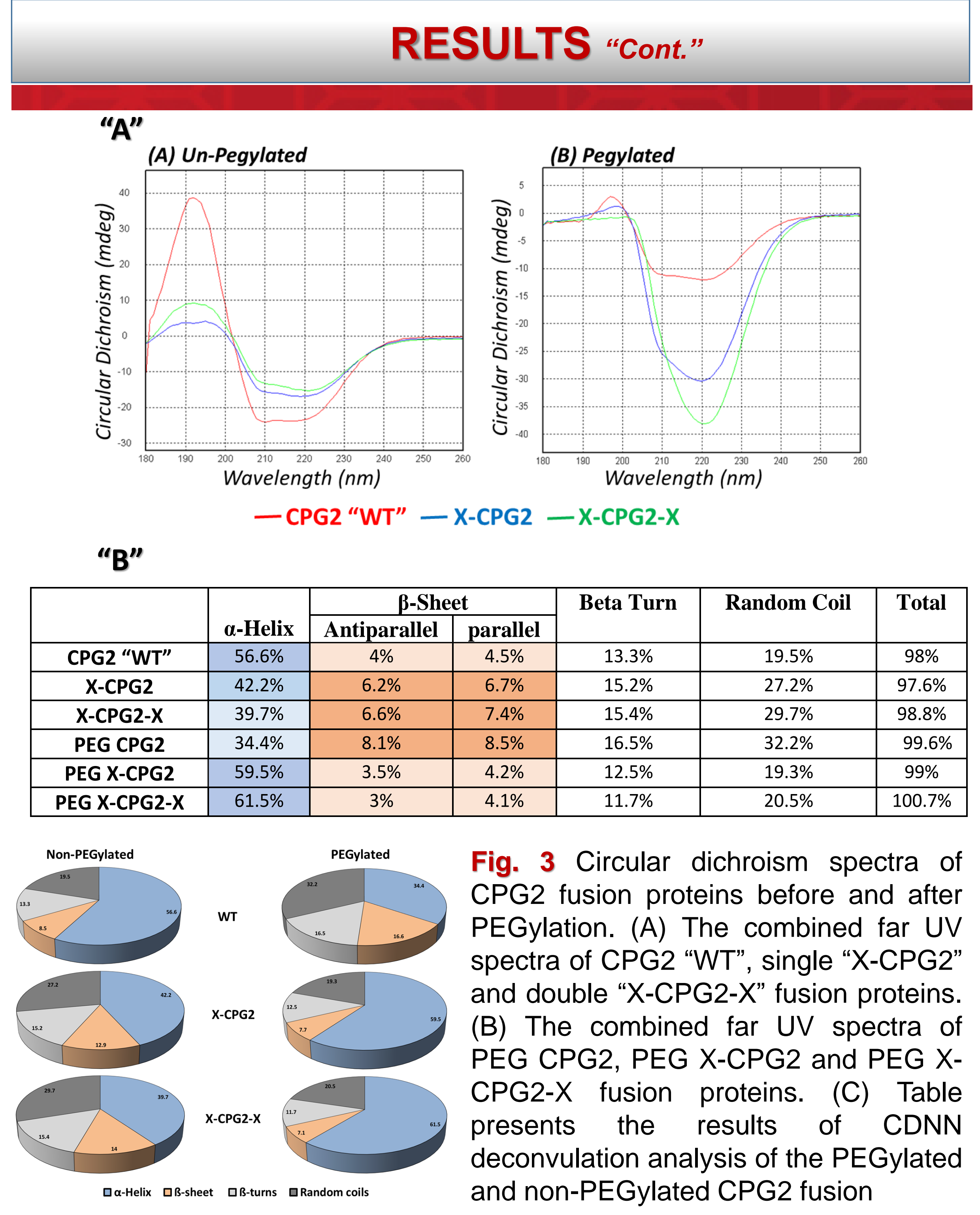

proteins $\mathrm{CD}$ spectra, showing their secondary structure composition. Darke shades indicate an increase in the composition percentage.

Enhanced CPG2 enzymatic activity and stability of the PEG CPG2 fusion proteins:

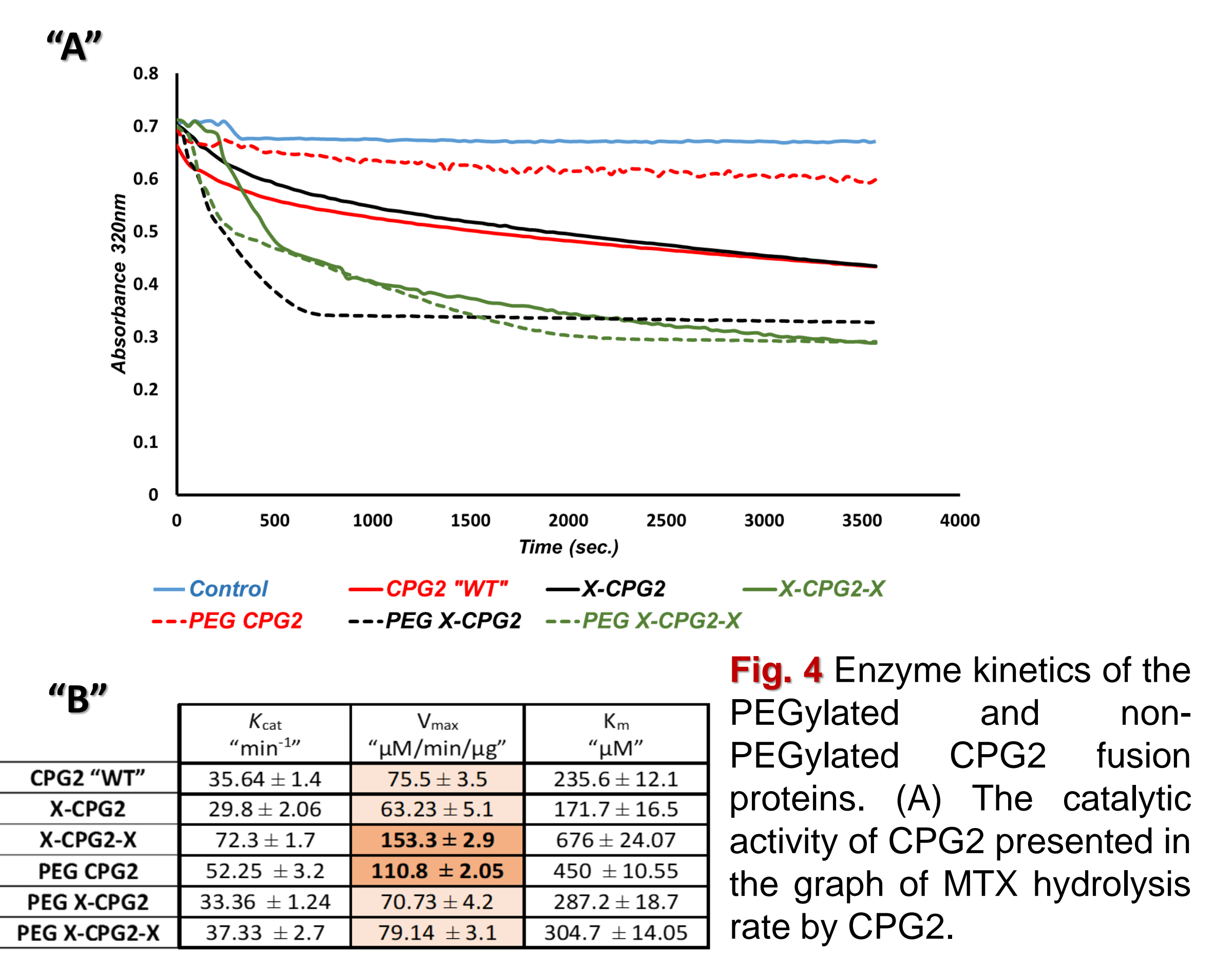

(B) The enzyme kinetics parameters ( $\mathrm{Km}, \mathrm{Vmax}$ and $\mathrm{Kcat})$ of the PEGylated and non-PEGylated CPG2 fusion proteins, Graph pad PRISM 6

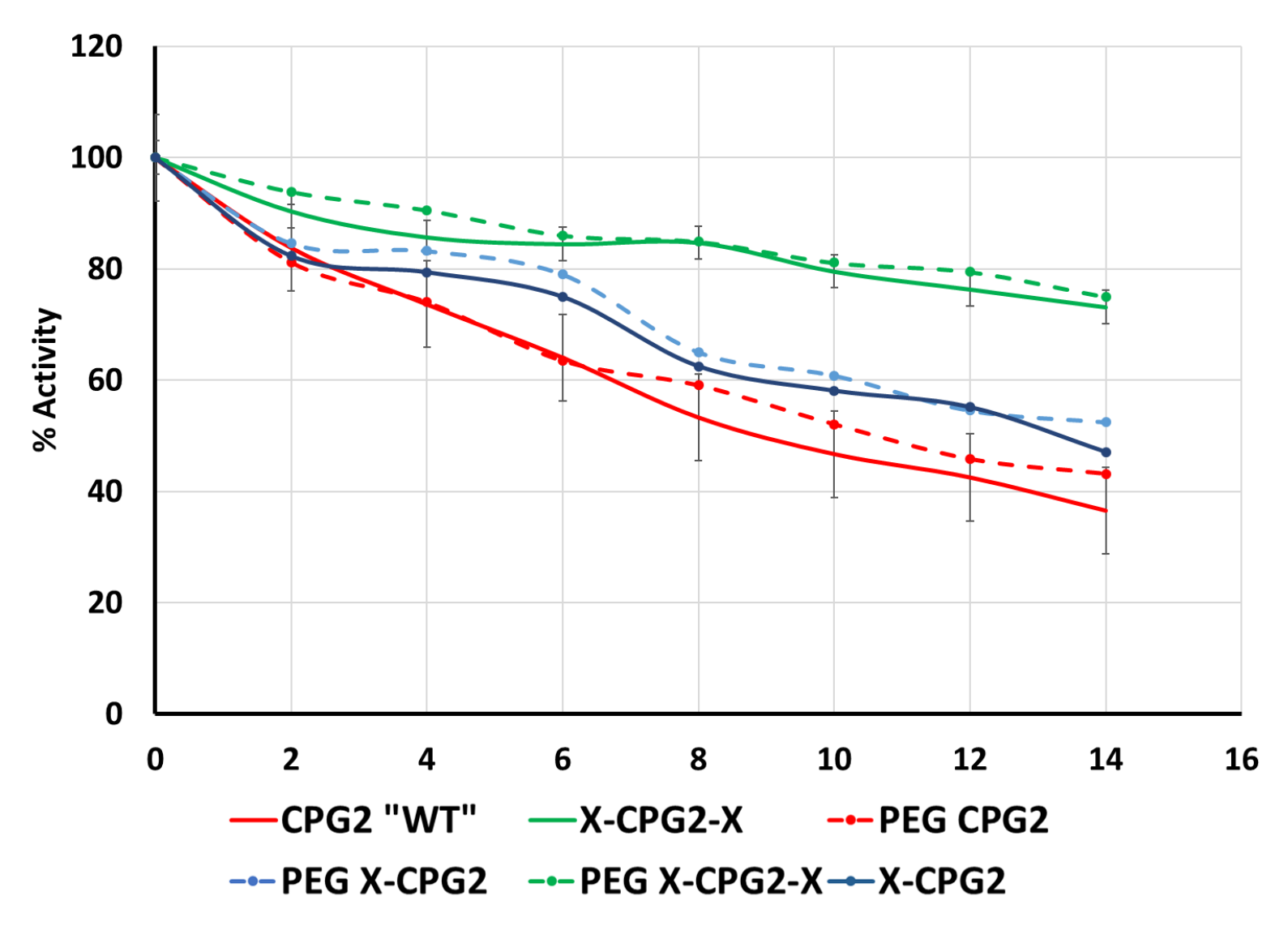

Fig. 5 PEGylated CPG2 fusion proteins stability in human plasma. Plasma samples from healthy donors were incubated at $37^{\circ} \mathrm{C}$ with $0.1 \mu / / \mu \mathrm{g}$ of the punzed PEGylatic (MTX hydrolytic) activity of CPG2 in each sample was tested every $48 \mathrm{hrs}$, and the percentage of remaining catalytic activity was plotted following normalization to a $100 \%$ activity at 0 time point.

Lowered Ex-vivo immunotoxicity of the PEGylated CPG2 fusion proteins.

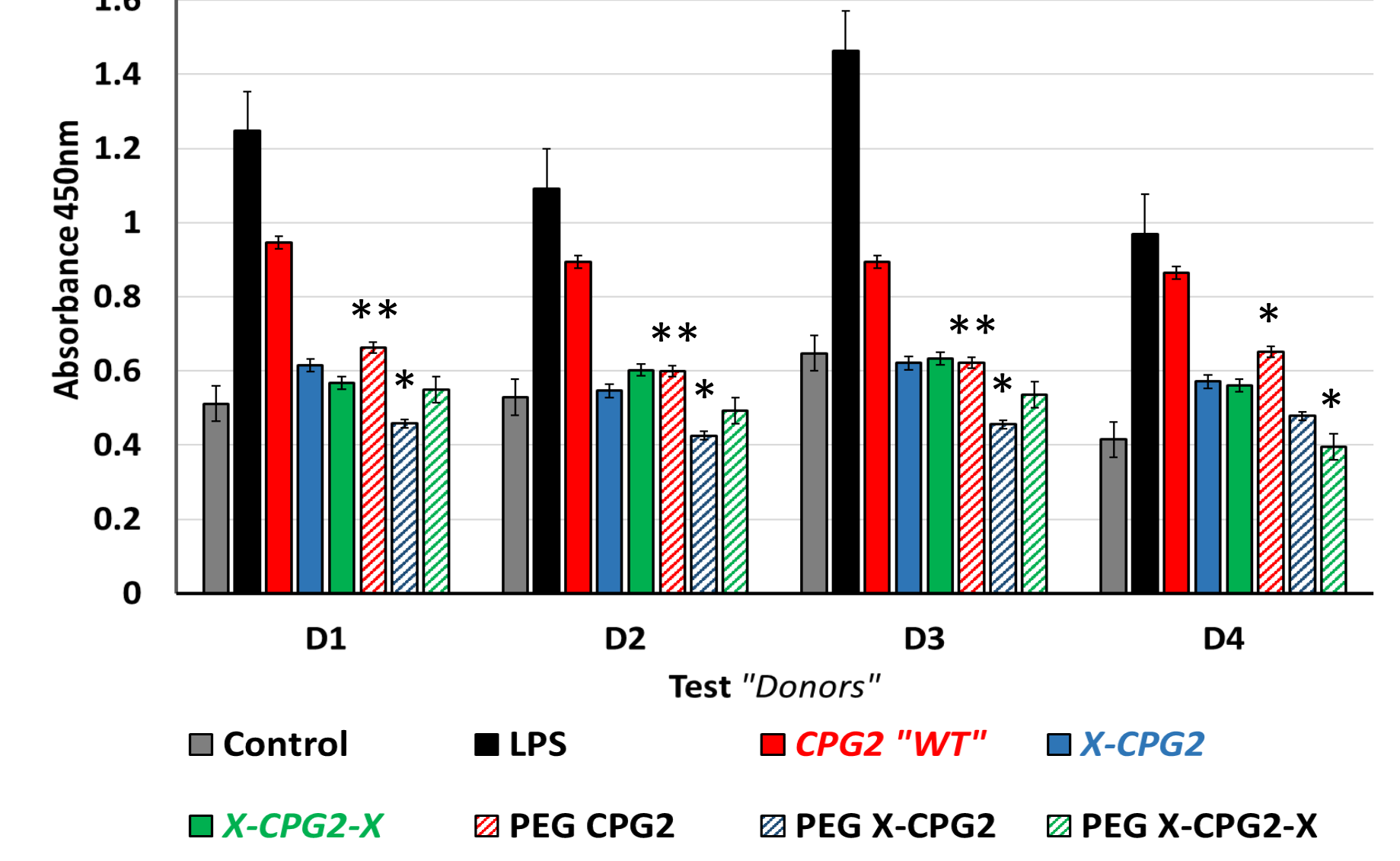

Fig. 6 Ex-vivo T cell proliferation assay. The immunotoxicity of PEGylated and non-PEGylated CPG2 fusion proteins was studied. PBMCs from "10ug/ml", in addition to the vehicle (PBS) as negative control and Lipolysaccharide "LPS" as positive control. After 48hrs cells incubated with the resultingCCK-8 solution for $4 \mathrm{hrs}$, and the resulting absorbance at $450 \mathrm{~nm}$ plotted for each donor (D).
RESULTS “Cont.

Differential cellular binding of the PEGylated CPG2 fusion protein in vitro:

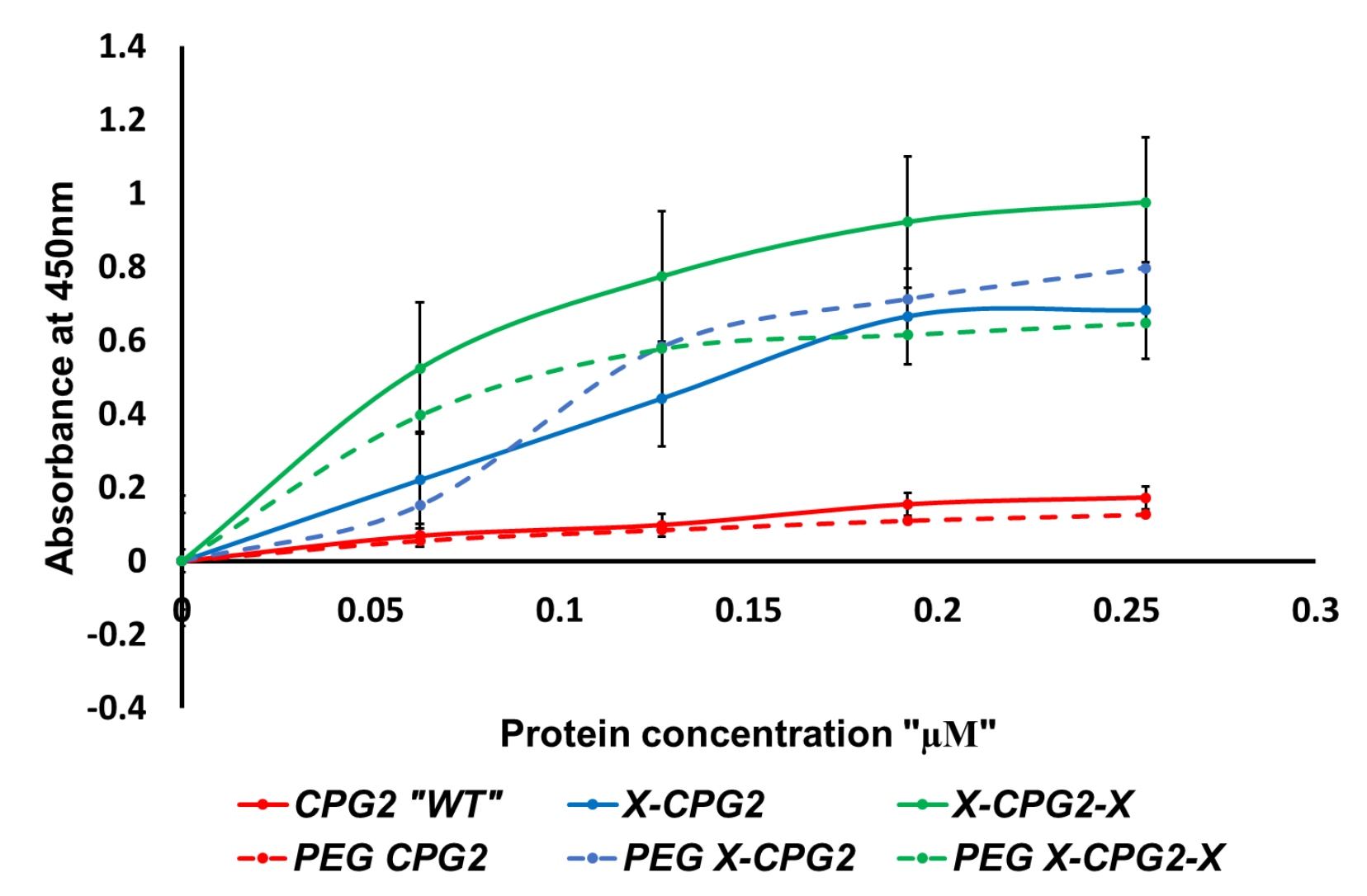

Fig. 7 In vitro binding strength of PEGylated and non-PEGylated CPG2 fusion proteins to the APN expressing cancer cell lines. low "A549" and high "HT1080 APN expressing cancer cell lines were cultured in 96-well plate with either PBS (as negative control) or increasing concentrations of the purified PEGylated and non-PEGylated CPG2 fusion proteins $(0.063$, $0.127,0.192$ and $0.255 \mu M)$. ELISA assay uning horseradish peroxidase capacities of the PEGylated fusion proteins.

Varied cytotoxicity of Methotrexate and the prodrug ZD2767P on cancer cell lines pre-treated with PEGylated CPG2 fusion proteins.
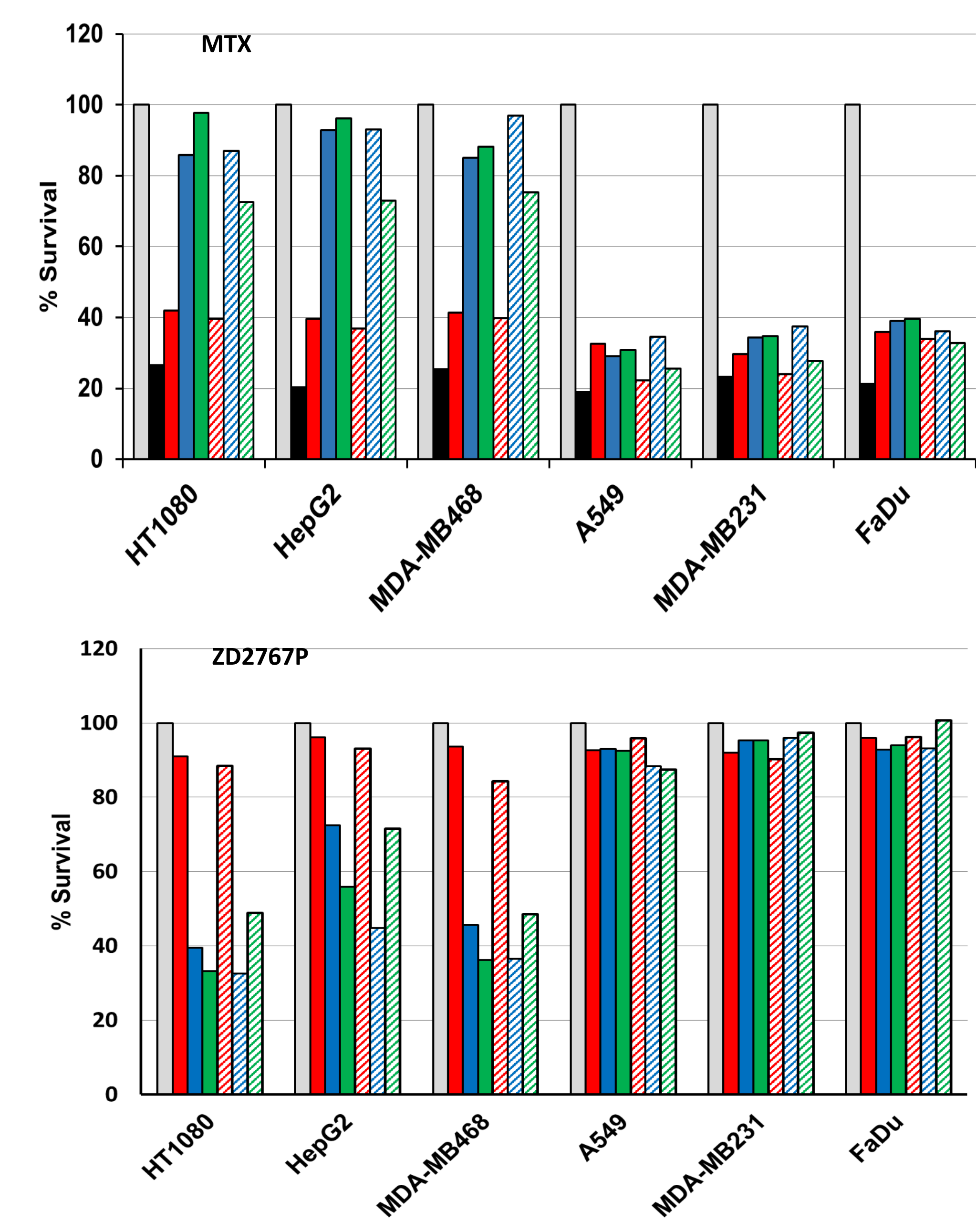

Fig. 6 The effect of Methotrexate (MTX) and the prodrug ZD2767P on cell viability following treatment with PEGylated and non- PEGylated (A 549 MAAB231 and FaDU) APN expressing cancer cell lines were (A) treatment with MTX and ZD2767P along with PBS "negative control" and MTX only "positive control for 48hrs. MTT assay was employed to obtain the percentage of cell viability. The resulting survival results are ormalized to the negative control as $100 \%$ surviva.

\section{CONCLUSION}

PEGylation of CNGRC-CPG2 fusion proteins and purification.

Structural analysis of the resulting PEGylation proteins showed a significant effect of PEGylation on alpha helix and beta sheet composition.

From our present results of lowered binding and activity of the PEGylated double fusion proteins, this was expected and probably steric hindrance and the PEG polymer conjugation sites lowered the enzyme substrate binding leading to lowered activity, moreover the receptor-peptide (APN-CNGRC) binding was similarly affected in case of

PEGylated double fusion proteins.

\section{REFERENCES}

- Carter PJ. Exp Cell Res. 2011;317(9):1261-9.

- Dozier JK, Distefano MD. International journal of molecula sciences. 2015;16(10):25831-64

3- Turecek PL, Bossard MJ, Schoetens F, Ivens IA. Journal of pharmaceutical sciences. 2016;105(2):460-75.

4- Mishra P, Nayak B, Dey RK. Asian Journal of Pharmaceutical Sciences. 2016;11(3):337-48.

5- AlQahtani AD, Al-Mansoori L, Bashraheel SS, Rashidi FB, AlYafei A, Elsinga P, Goda SK. Eur J Pharm Sci. 2019;127:79-91.

6- Al-mansoori L, Bashraheel SS, Qahtani ADA, O'Connor CD, Elsinga P, Goda SK. Oncotarget. 2020;11:619-633. 